\title{
Mixed (1D-2D) quantum electron transport in percolating gold film
}

\author{
E. Yu. Beliayev, B. I. Belevtsev* and Yu. A. Kolesnichenko \\ B. Verkin Institute for Low Temperature Physics and Engineering, \\ National Academy of Sciences, Kharkov 61103, Ukraine
}

\begin{abstract}
The gold film (mean thickness $\approx 3.5 \mathrm{~nm}$ ) was condensed in high vacuum at temperature 70 K on single-crystal sapphire substrate. The transport properties of the film at low temperature reveal simultaneously indications of $1 \mathrm{D}$ and $2 \mathrm{D}$ quantum interference effects of weak localization and electron-electron interaction. It is shown that this behavior is determined by inhomogeneous electron transport at the threshold of thickness-controlled metal-insulator transition.
\end{abstract}

PACS numbers: 73.20.Fz, 73.50.Jt, 71.30.+h 


\section{INTRODUCTION}

Metal-insulator transition (MIT) in disordered systems is still a basic challenge in the solid-state physics. One of the principal causes of MIT is a sufficient increase in lattice disorder (so called Anderson transition). It was theoretically stated that a two-dimensional (2D) system of non-interacting electrons should be insolating at any degree of disorder when going to zero temperature [1]. Electron-electron interaction plays, however, crucial role in 2D transport especially at low temperature [2 4], so that MIT in 2D systems is asserted theoretically as possible which finds also some experimental support [2, 3]. Two types of crystal-lattice disorder affecting electron propagation in conducting solids are usually considered. The first is associated with perturbations of the scattering potentials on the atomic scale: impurities, vacancies, and so on, and is often called microscopic. At the same time, MIT can also take place in inhomogeneous systems such as disordered mixtures of a metal and an insulator, granular metals, and the like [5, 6], where the scale of disorder is well above interatomic distances, and where propagating electrons should overcome insulating regions (or boundaries) between the metallic clusters (grains). This second type of disorder is called macroscopic. MIT in systems with macroscopic disorder is inevitably associated with (classical or quantum) percolation effects in electron transport.

A good few known experimental studies of MIT have been performed with ultrathin metal films by means of step-by-step increasing of the film thickness (see reviews [3, 5] and references therein). The nominal thickness increments at these studies are usually in the range 0.05-0.1 $\AA$, that is much less than interatomic distances. This brings the questions: are the films remain homogeneous in thickness at these small increments, and are the films homogeneous at all at MIT, which occurs at some critical thickness, $d_{c}$ ? It is known from available literature about this type of MIT studies, that $d_{c}$ is in the range $1-5 \mathrm{~nm}$ depending on film structure (crystalline or amorphous) and material of substrate. The minimum values of $d_{c}(1-1.5 \mathrm{~nm})$ were found in quench-condensed amorphous Bi and Ga films with thin underlayer of amorphous Ge [7]. For the most cases the sheet resistance of films, $R_{\square}$, with thickness $d \approx d_{c}$ is order of $10 \mathrm{k} \Omega$, which is comparable with the resistance quantum $R_{Q}=R_{\square}=a \hbar / e^{2}$, where $\hbar / e^{2} \approx 4.1 \mathrm{k} \Omega$ and $a$ is order of unity. $R_{Q}$ is often considered as some characteristic resistance for 2D MIT. It is evident that condensed films with $R_{\square} \geq R_{Q}$ are discontinuous, so that percolating effects should be important. 
In this study, we present transport properties of quench-condensed gold film with thickness about $3.56 \mathrm{~nm}$ and $R_{\square}$ about $5 \mathrm{k} \Omega$ above $10 \mathrm{~K}$. This film has weak nonmetallic temperature dependence of resistance with logarithmic behavior above $10 \mathrm{~K}$ and somewhat stronger dependence at low temperature. The film is characterized by quantum interference effects in electron transport. Above $3 \mathrm{~K}$ only 2D effects in transport have been found; whereas, below $3 \mathrm{~K}$ both one-dimensional (1D) and 2D effects in transport properties can be distinguished. This reflects inhomogeneous structure (and corresponding electron transport) of the film near the thickness-controlled MIT in ultrathin metallic films.

\section{EXPERIMENTAL TECHNIQUE}

The gold film studied has been prepared by thermal evaporation from a Mo boat onto a substrate of polished single-crystal sapphire plate. The size of the film regions being measured was $2 \times 0.1 \mathrm{~mm}^{2}$. The substrate was preliminarily etched in hot aqua regia, then washed in distilled water and etched in boiling bichromate, then again washed in distilled water, and finally dried in dessicator.

Deposition of the film on the prepared in this way substrate and subsequent measurement in situ its transport properties were carried out in the high-vacuum cryostat containing ${ }^{3} \mathrm{He}$ and a superconducting solenoid. Oil-free vacuum pumps were used to reach pressure about $1 \times 10^{-7} \mathrm{~Pa}$. A system of sliding screens (masks) was used for deposition of gold contacts (at room temperature) and subsequent deposition of the gold film (at $T \approx 70 \mathrm{~K}$ with the rate $0.05 \mathrm{~nm} / \mathrm{s}$ ). Initial purity of gold deposited was 99.99\%. Pressure of residual gas during deposition was about $5 \times 10^{-6} \mathrm{~Pa}$. Resistance of the film during its growth has been followed (under applied voltage $U_{\text {appl }}=1 \mathrm{~V}$ ), and deposition was interrupted when

$R_{\square}$ had reached about $4.4 \mathrm{k} \Omega$. According to our previous studies [8], structure of gold films with $R_{\square}$ about $5 \mathrm{k} \Omega$ deposited at similar conditions is close to percolation threshold. The nominal film thickness, determined by a quartz sensor, is $3.56 \mathrm{~nm}$. The sample prepared was held at the preparation temperature $(70 \mathrm{~K})$ during $12 \mathrm{~h}$ up to stopping of small resistance variation due to structural relaxation inherent for films deposited on cold substrates. The magnetoresistive measurements were carried out in situ in lower temperature range $0.4-30 \mathrm{~K}$ in magnetic fields from -0.05 to $+5 \mathrm{~T}$. The resistance was determined by measuring current in conditions of preset applied voltage $U_{a p p l}$. The most of measurements have been done for 
$U_{a p p l}=0.2,1$ and $5 \mathrm{~V}$, although other voltages were also used, especially for determination of the current-voltage characteristic at different temperatures (see below).

\section{RESULTS AND DISCUSSION}

\section{General characterization of transport properties of the film}

It is found that conducting state of the film studied corresponds to that on the threshold of the thickness controlled MIT. Temperature dependence of resistance has nonmetallic $(d R / d T<0)$ character, which above $10 \mathrm{~K}$ and at high enough applied voltage follows the logarithmic law $(\Delta R \propto \ln T)$ (Fig. 1). In the low temperature range (below $5 \mathrm{~K}$ ), the explicit dependence of resistance on applied voltage takes place (Figs. 1 and 2). It is seen (Fig. 2) that at $T=5 \mathrm{~K}$ a decrease in voltage leads only to a slight increase in resistance so that at this temperature (and above it) Ohm's law is obeyed to a good approximation. At lower temperature, however, the exponential increase in resistance with decreasing voltage is seen in low-voltage range $\left(U_{a p p l}<50 \mathrm{mV}\right)$ (inset in Fig. 2), although for $U_{a p p l} \geq 2 \mathrm{~V}$ the non-Ohmic behavior is pronounced insignificantly. It should be noted that the same voltage dependence $\left[R \propto \exp \left(1 / U_{\text {appl }}\right)\right]$ in low-temperature range (but with far larger amplitude in $R$ variations) has been observed in Ref. [8] in percolating gold film obtained under the same conditions as in this study. But being with less nominal thickness $(3.25 \mathrm{~nm})$ the film in Ref. [8] was accordingly more resistive and demonstrated a transition from strong to weak

electron localization under increasing $U_{\text {appl }}$ in full measure; whereas, in the film studied only some indications of such type of behavior can be found.

Thus the transport properties testify to inhomogeneous structure of the film. This is sure connected with spatial inhomogeneity in thickness. The small effective thickness of the film $\left(3.56 \mathrm{~nm}\right.$ ), high values of $R_{\square}$ (about $6 \mathrm{k} \Omega$ and higher) and rather strongly pronounced nonohmic behavior of its conductivity in low temperature range indicate that the film is partially discontinuous. This means that it consists of metallic islands (or percolating clusters) separated by tunnel barriers. Two types of barriers are possible: (a) vacuum gaps between adjacent islands on an insulating substrate, and (b) narrow and thin constrictions (bridges) between islands in the case of weak contacts between neighboring islands. In any case, such a system can be regarded as similar to a two-dimensional granular metal. These 
suggestions are fully in line with the results of known studies of quench-condensed (substrate temperature between $4 \mathrm{~K}$ and $77 \mathrm{~K}$ ) Au films [9]. It was found in particular [9] that the critical nominal thickness, $d_{c r}$, marking the onset of conductivity is about $2 \mathrm{~nm}$ for quenchcondensed Au films that agrees reasonably well with results of this study. It follows from results of Ref. [9] that quench-condensed Au films deposited on a weak-binding substrate (like that in this study) form a 2D disordered array of weak-connected islands at thicknesses moderately greater than $d_{c r}$. The grain diameter, $d_{G}$, in this type of Au film is in the range $10 \mathrm{~nm}<d_{G}<20 \mathrm{~nm}[9]$.

A quasi-granular 2D structure of the film is also supported by the following consideration. It is known that product $\rho l\left[\right.$ where $\rho\left(=R_{\square} d\right)$ and $l$ are the resistivity and the mean-free path, respectively] is characteristic constant for typical metals. For gold, $\rho l \approx 8.43 \times 10^{-16} \Omega \mathrm{m}^{2}$ can be found in the framework of the quasi-free electron approximation. Taking this relation, $l \approx 0.05 \mathrm{~nm}$ can be obtained for the film studied. So low nominal value (less than interatomic distance) indicates clearly an inhomogeneous structure of the film studied on the threshold of the thickness controlled MIT. The high resistivity is determined by low intergrain tunnel conductivity; whereas, intragrain conductivity can be far larger. We will take into account the particular morphology of the quench-condensed ultrathin gold films at discussion of the results obtained.

\section{Quantum interference effects in conductivity of the film studied}

The logarithmic law $(\Delta R \propto \ln T)$ found in thin film studied (Fig. 1) implies that it is connected with quantum weak localization (WL) and electron-electron interaction (EEI) effects in conductivity of 2D systems [4]. The two-dimensional conditions of manifestation of these effects are: $d<L_{\varphi}, L_{T}$, where $d$ is the film thickness, $L_{\varphi}=\left(D \tau_{\varphi}\right)^{1 / 2}$ is the diffusion length of phase relaxation, $L_{T}=(\hbar D / k T)^{1 / 2}$ is the thermal coherence length in a normal metal, $D$ is the electron diffusion coefficient, and $\tau_{\varphi}$ is the time of phase relaxation. The lengths $L_{\varphi}$ and $L_{T}$ are attributed to the WL and EEI effects, respectively. Macroscopic disorder (percolating and/or granular structures) can induce a dramatic influence on the WL and EEI effects [10] up to their total depression at strong enough disorder. At the same time, a rather weak macroscopic disorder has no significant influence on these quantum

effects. In particular, a system behaves as homogeneous in respect to WL and EEI effects if 
the relevant lengths $\left[L_{\varphi}(T)\right.$ and $\left.L_{T}\right]$ are larger than characteristic inhomogeneity scale (for example, percolation correlation length, $\xi_{p}$, or grain diameter, $d_{G}$, in 2D granular metal).

For homogeneous 2D system the contribution of WL and EEI to the temperature dependence of conductivity in zero magnetic field is given by [4]

$$
\Delta \sigma(T)=\frac{e^{2}}{2 \pi^{2} \hbar}\left\{-\left[\frac{3}{2} \ln \frac{\tau_{\varphi}^{*}}{\tau}-\frac{1}{2} \ln \frac{\tau_{\varphi}}{\tau}\right]+\lambda_{T}^{D} \ln \frac{k T \tau}{\hbar}\right\}
$$

where $\tau$ is the elastic electron relaxation time, $\tau_{\varphi}^{-1}=\tau_{i n}^{-1}+2 \tau_{s}^{-1} ;\left(\tau_{\varphi}^{*}\right)^{-1}=\tau_{i n}^{-1}+(4 / 3) \tau_{s o}^{-1}+$ $(2 / 3) \tau_{s}^{-1}, \tau_{i n}$ is the phase relaxation time due to inelastic scattering, $\tau_{s o}$ and $\tau_{s}$ are spin relaxation times of electrons due to spin-orbit and spin-spin scattering, respectively. $\lambda_{T}^{D}$ is the interaction constant in the diffusive channel, which is close to unity for typical metals. The time $\tau_{i n}$ is temperature dependent, namely, $\tau_{i n}^{-1} \propto T^{p}$, where $p$ is the exponent which depends on the mechanism of inelastic scattering. The first term in Eq.(1) corresponds to WL effects, while the second one to EEI.

The Eq. (11) presents well known logarithmic correction to conductivity which can be rewritten as [4]

$$
\frac{\Delta R}{R}=-a_{T} \frac{e^{2} R_{\square}^{e f f}}{2 \pi^{2} \hbar} \ln \left(\frac{k T \tau}{\hbar}\right),
$$

with $a_{T}$ order of unity, exact value of which is determined by dominating mechanisms of phase relaxation in a specific system.

It is known that gold is characterized by strong spin-orbit interaction $\left[\tau_{s o} \ll \tau_{i n}(T)\right]$, which determines specific value of $a_{T}$, and causes appearance of positive magnetoresistance due to the WL effect [4], as it is usually observed in gold films (Refs. [8, 11] and Refs. therein), including the film studied (see Fig. 1 and more detailed information below). From Eq. (1), on conditions of strong spin-orbit scattering and neglecting the spin-spin interaction $\left[\tau_{s o} \ll \tau_{i n}(T)\right.$ and $\left.\tau_{s} \gg \tau_{s o}, \tau_{i n}\right]$, it can be obtained that $a_{T}=1-p / 2$, where unity comes from the EEI effect and second part is determined by the WL effect with $p$ being the exponent in temperature dependence $\tau_{i n}^{-1} \propto T^{p}$ for a dominant mechanism of phase relaxation.

Generally, the phase relaxation is determined by the two main contributions: electronelectron and electron-phonon interactions, so that $\tau_{i n}^{-1}=\tau_{e e}^{-1}+\tau_{e p}^{-1}$, where $\tau_{e e}$ and $\tau_{e p}$ are the corresponding times. It was found for the electron-phonon interaction processes in disordered $\left(R_{\square}\right.$ up to $\left.\approx 500 \Omega\right)$ gold films that $\tau_{e p}^{-1} \propto T^{p}$ with exponent $p$ between 2 and 2.8 [11]. According to Ref. [11], the electron-phonon scattering dominates the rate of phase relaxation in gold films only above $10 \mathrm{~K}$. Below this temperature $\tau_{i n}^{-1} \propto T$ was found and 
attributed to influence of electron-electron scattering. It is known that the phase relaxation rate due to electron-electron scattering in 2D systems is determined by collisions with small energy transfer $(\Delta E \ll k T)[4]$

$$
\tau_{e e}^{-1}(T)=\frac{\pi k T}{\hbar} \frac{e^{2} R_{\square}}{2 \pi^{2} \hbar} \ln \left(\frac{\pi \hbar}{e^{2} R_{\square}}\right)
$$

Increasing disorder leads to domination of EEI in the phase relaxation (that is $\tau_{e e}^{-1} \gg \tau_{e p}^{-1}$ ). Since the film studied is far more disordered than those in Ref. [11] the temperature range of EEI domination in it should be much wider. In this case, $p=1$ should be taken and, consequently, $a_{T}=1 / 2$ is to expect.

Now let us consider experimental values of $a_{T}$. It is found in the film studied that $a_{T} \approx 1.15$ at $U_{a p p l}=0.2 \mathrm{~V}$ at $T>8 \mathrm{~K}$ and it is close to unity for higher voltage in the range above $5 \mathrm{~K}$ (Figs. 1 and 3). When using Eq. (2) for deriving $a_{T}$ values we have taken the measured macroscopic resistance $R_{\square}^{e x p}$ as $R_{\square}^{e f f}$. This may be done only for homogeneous systems. If a system can be supposed to be inhomogeneous, $R_{\square}^{e f f}$ should be a fitting parameter [12]. We have checked out this matter. For instance, from analysis of logarithmic $R(T)$ dependence at $U_{a p p l}=5 \mathrm{~V}$ we found that $R_{\square}^{e f f}=5.28 \mathrm{k} \Omega$ given $a_{T}=1$. This sheet resistance value falls within range of the $R_{\square}$ temperature variations at $U_{\text {appl }}=5 \mathrm{~V}$ (Fig. 1), so that at this voltage the system behaves as homogeneous one above $5 \mathrm{~K}$.

The derived values of $a_{T} \approx 1$ do not correspond to $a_{T} \approx 1 / 2$ expected for systems with strong spin-orbit interaction. So it looks like that only EEI makes contribution to logarithmic $R(T)$ dependence. A rather plain suggestion could be that in the film studied the WL correction is significantly suppressed for some reasons so that EEI contribution has dominant effect on $R(T)$ which results in $a_{T} \approx 1$. The total suppression of WL is possible in some cases, for example, by application of strong enough magnetic field $\left(H>H_{\varphi}=\hbar c / 4 e L_{\varphi}^{2}\right)[4]$. As it is seen from Fig. 1, the application of field $H=4.2 \mathrm{~T}$, which is far larger than $H_{\varphi}$ (as will be clear below) causes only weak effect on a slope of the linear part of dependence $\Delta R v s$ $\ln T$ above $T \approx 8 \mathrm{~K}$, so that it seems that WL is significantly suppressed in the film even in zero field. This situation is possible in inhomogeneous (percolating or granular) 2D films [6]. In a system consisting of rather large grains separated by weak tunnel barriers appearance of the WL correction (same as in an homogeneous system) is determined by closed electron trajectories with self-intersections that increases the probability of back-scattering [4]. The size of these trajectories is about $L_{\varphi}$. The EEI correction is determined by length $L_{T}$. 
Generally, in the case of quasi-particle description of electrons, the relation $L_{T} \ll L_{\varphi}$ holds [4]. In any case, for the WL and EEI corrections to be clearly seen in $R(T)$ dependences, the both lengths $L_{\varphi}$ and $L_{T}$ should exceed the characteristic inhomogeneity scale of the system (for example, grain size $d_{G}$ ). Since $L_{\varphi} \gg L_{T}$, electron trajectories which determine WL effect should include more grains (and more intersections with grain boundary) than those connected with EEI effect, so that weak intergrain connections can induce more depressing effect on WL correction than that on EEI one.

It is seen in Fig. 1 that $R(T)$ follows the logarithmic law only for high enough temperature. $R(T)$ begins to deviate from $\ln T$ behavior below $5 \mathrm{~K}$ at $U_{\text {appl }}=5 \mathrm{~V}$, and seems to go to saturation at low enough temperature. In this case an overheating effect cannot be excluded. The overheating should be, however, diminished with decreasing voltage. Really, the saturation has disappeared at $U_{a p p l}=1 \mathrm{~V}$, and for the lower voltage $U_{a p p l}=0.2 \mathrm{~V}$ even an increased rate of the $R(T)$ growth with decreasing temperature is observed in low temperature range (Fig. 1). The latter case is presented more clearly in Fig. 3. Below $9 \mathrm{~K}$ a deviation from $\ln T$-law appears, which is presented in the inset as $\Delta R=f\left(T^{-1 / 2}\right)$. This will be discussed in detail below.

The magnetoresistance (MR) data obtained reflect inhomogeneous structure of the film studied as well. General view of MR curves and their variations with temperature can be seen in Fig. 4. Except for low-field $(H \leq 0.02 \mathrm{~T})$ and low-temperature $(T \leq 3.1 \mathrm{~K})$ ranges (see below), the magnetoresistive curves are found to correspond to the known expression describing MR due to WL effect for 2D systems [4]:

$$
\Delta \sigma(H)=\frac{e^{2}}{2 \pi^{2} \hbar}\left\{\frac{3}{2} f_{2}\left(\frac{4 e D H \tau_{\varphi}^{*}}{\hbar c}\right)-\frac{1}{2} f_{2}\left(\frac{4 e D H \tau_{\varphi}}{\hbar c}\right)\right\}
$$

where $f_{2}(x)=\ln (x)+\Psi(1 / 2+1 / x), \Psi$ is digamma function. $f_{2}(x) \approx x^{2} / 24$ for $x \ll 1$, and $f_{2}(x) \propto \ln x$ for $x \gg 1$. For the films studied, the contribution of EEI to MR is negligible, so we do not cite corresponding expressions.

Positive MR found (Fig. 4) is expected for gold films with strong spin-orbit scattering. For this case $\left[\tau_{s o} \ll \tau_{i n}(T)\right]$, Eq. (44) can be rewritten as [4]

$$
\frac{\Delta R(H)}{R}=R_{\square}^{M R} \frac{e^{2}}{4 \pi^{2} \hbar} f_{2}\left(\frac{4 e H L_{\varphi}^{2}}{\hbar c}\right) .
$$

We have found that Eq. (5) describes adequately the experimental $R(H)$ dependences for $H \leq 1 \mathrm{~T}$ with small deviations for higher field. The Eq. (4) describes well experimental 
$R(H)$ in the whole field range, and the both equations have been used to derive the $L_{\varphi}(T)$ dependence.

For homogeneous systems, measured macroscopic resistance $R_{\square}^{e x p}$ can be substituted for $R_{\square}^{M R}$ when processing experimental data by Eqs. 4 or 5. $R_{\square}^{M R}$ is resistance on the scale $L_{\varphi}$, which in an inhomogeneous sample can be less than macroscopic resistance measured on much larger scale. In this case, $R_{\square}^{M R}$ should be a fitting parameter [12, 13]. We have really found that computed values of $R_{\square}^{M R}$ are somewhat less than $R_{\square}^{e x p}$ (on about 20\%). This difference is not, however, crucial and we have not found a significant effect of it on calculated values of $L_{\varphi}$.

The temperature dependence of $L_{\varphi}(T)$ in the range $1.4-30 \mathrm{~K}$ is shown in Fig. 5. It is seen that generally $L_{\varphi}(T)$ is not essentially dependent on the applied voltage. In particular, in the range $3-10 \mathrm{~K}$, the data for different $U_{\text {appl }}$ practically coincide. Above $T \approx 10 \mathrm{~K}$ the data become more noisy due to smaller magnitude of $\mathrm{MR}$ in this range. In low-temperature range (below $3 \mathrm{~K}$ ), influence of $U_{a p p l}$ on $L_{\varphi}$ is evident. In this range the $L_{\varphi}(T)$ values tend to saturation with decreasing temperature below $3 \mathrm{~K}$. The following temperature dependences $L_{\varphi}(T)$ were found: $L_{\varphi}(T) \propto T^{-0.35}(3-15 \mathrm{~K})$ and $L_{\varphi}(T) \propto T^{-1.05}(15-30 \mathrm{~K})$. Since $L_{\varphi} \propto$ $T^{-p / 2}$, the first case corresponds to $p \approx 0.7$ and the second to $p \approx 2.1$. We will stop at these features of $L_{\varphi}(T)$ in the next section.

\section{Enhancement of percolating character of electron transport at low temperature} and $1 \mathrm{D}$ effects in the film conductivity

In low temperature range we have found a weak but quite distinct anomaly of MR behavior in low-field range, which is shown on a large scale in Fig. 6. This anomaly disappears with increasing field and is depressed with increase in temperature up to $T \simeq 3.1 \mathrm{~K}$ (Fig. 7). The field at which the MR anomaly comes to saturation (and to transition to 2D MR behavior) increases with decreasing temperature being in the range 0.01-0.03 $\mathrm{T}$ (Fig. 7). At low field $(H \leq 0.004 \mathrm{~T}) \mathrm{MR}$ in the range of this anomaly follows the quadratic law $\Delta R(H) / R(0) \propto H^{2}$ (Fig. 8). Temperature behavior of maximum MR of the anomaly (at field position of saturation) is shown in the inset of Fig. 8.

It should be noted that this type of anomaly have been seen by us more than once at low enough temperature in other fairly disordered gold films (close to the thickness controlled 
percolation threshold). Results obtained permit us to assert that this low-temperature MR anomaly together with the above-mentioned deviations of $R(T)$ from logarithmic behavior at low temperature range (Fig. 3) are indications of 1D effects in film conductivity caused by inhomogeneous (granular) film structure near the percolation threshold. Indications of mixed 1D-2D conductivity in percolating gold films were first suggested to be seen in Ref. [10]. Further 1D interference effects had been studied in specially made narrow enough films (see [12, 14 16] and references therein). The theory of quantum interference (WL and EEI) effects in 1D conductors has been developed in Refs. [4, 17, 18].

Before doing further analysis and discussion of the 1D effects in the film studied, the principal causes of appearance of these effects in ultrathin films should be considered. As was mentioned above, a film near the thickness-controlled percolation threshold consists of weakly connected islands (grains). The intergrain connections are determined by narrow constrictions, which can make tunnel junctions (like point contacts). In real quasi-island films near the percolation threshold the intergrain constrictions (contacts) are not the same throughout the system, thus, the conductivity is percolating. It is determined by the presence of optimal chains of grains with maximum probability of tunnelling for adjacent pairs of grains forming the chain. At low enough temperature the tunnelling can become activated. In conditions of activated conductivity, the number of conducting chains decreases with decreasing temperature, so that at low enough temperature a percolation network can consist of a few conducting channels or even come to a single conducting path [19]. It appears to be a general trend for disordered systems, and in particular for 2D systems. For example, it was theoretically shown [20], that increasing in disorder in 2D system leads to forming of a narrow channel along which electrons propagate through a disordered sample.

It follows from the aforesaid that thin enough percolating film can manifest a mixed (1D and 2D) behavior of the interference effects in the conductivity. In this case, similar as in Ref. [10], it can be suggested that quantum correction to conductivity has two contributions,

$$
\frac{\Delta R(T, H)}{R}=\left(\frac{\Delta R(T, H)}{R}\right)_{1 D}+\left(\frac{\Delta R(T, H)}{R}\right)_{2 D}
$$

where lower indices $1 D$ and $2 D$ mark the corresponding contributions. Relative contribution of each term is temperature and magnetic-field dependent, so that, for example, the 1D term can even disappear at high enough $T$ or $H$.

Expressions for the 2D corrections have been presented above. For further discussion some 
valid 1D expressions should be considered. Conditions for appearance of 1D interference effects in electron transport are that both characteristic lengths, $L_{T}=(\hbar D / k T)^{1 / 2}$ and $L_{\varphi}=\left(D \tau_{\varphi}\right)^{1 / 2}$, must be greater than width, $W$, and thickness, $d$, of the $1 \mathrm{D}$ wire. It is known [4, 14, 16] that 1D effects become apparent only at low temperatures where the main contribution to the phase breaking gives the electron-electron scattering with small energy transfer [4, 17, 18]. This is, so called, Nyquist phase-breaking mechanism which is especially important for low-dimensional systems [4, 16, 18]. For 2D systems the Nyquist time is presented by Eq. (3). For 1D systems this time is denoted by $\tau_{N}$, and corresponding phase relaxation rate is given by [4, 18]

$$
\tau_{N}^{-1}=\left[\left(\frac{e^{2} R_{\square}}{\hbar}\right)\left(\frac{k T}{\hbar}\right)\left(\frac{\sqrt{2 D}}{W}\right)\right]^{2 / 3} \propto T^{2 / 3} .
$$

The characteristic phase-relaxation length for this process is $L_{N}=\left(D \tau_{N}\right)^{1 / 2} \propto T^{-1 / 3}$.

In line with our suggestion about mixed (1D and 2D) behavior of the film conductivity at low temperature we have compared the deviations of $R(T)$ from logarithmic $2 \mathrm{D}$ behavior at low temperature range (Fig. 3) with known 1D expressions. EEI correction to the resistance a single film strip (at $W<L_{T}$ ) is [4]

$$
\frac{\Delta R_{\text {int }}(T)}{R}=\frac{e^{2}}{2 \hbar} \frac{R_{\square}^{s t r}}{W} L_{T} \propto T^{-1 / 2},
$$

where $R_{\square}^{s t r}$ is sheet resistance of the strip. This quantum correction increases with decreasing temperature as $T^{-1 / 2}$, that agrees rather well with the $\Delta R(T)$ behavior shown in inset of Fig. 3. It should be noted that dependence $\Delta R \propto T^{-1 / 2}$ in the film studied takes place also at high magnetic field which depressed completely WL effect, so that this behavior should be attributed solely to EEI effect. Similar behavior corresponding to Eq. (8) has been previously seen in 1D Au films [12, 15].

Of course, WL effect can also influence quantum 1D transport [4, 18]. However, since gold is characterized by strong spin-orbit scattering (antilocalization), the corresponding correction, $\Delta R_{l o c}(T)$, is negative and $R$ should decrease with decreasing temperature. It was found in $1 \mathrm{D}$ gold films that at low enough temperature EEI effect gives dominating contribution to the quantum correction [12, 16]. Apparently this occurs in the gold film studied as well.

Consider now in more detail the low-field anomaly of MR in low-temperature range (Figs. 6 and 7), which we have attributed to 1D effects as well. MR of 1D systems in low magnetic 
field is determined exclusively by WL [4]. At low enough temperature the phase relaxation is determined by Nyquist mechanism so that $\tau_{N} \ll \tau_{\varphi 0}(H=0)$, where $\tau_{\varphi 0}$ is phase relaxation time due to all other phase-breaking mechanisms. In the case of strong spin-orbit scattering, WL correction to resistance of $1 \mathrm{D}$ film for magnetic field perpendicular to the film plane is [4, 16, 17]

$$
\frac{\Delta R_{l o c}(T, H)}{R_{0}}=-0.31 \frac{e^{2} R_{\square}^{s t r}}{\hbar W}\left[1 / L_{N}^{2}+1 / D \tau_{H}\right]^{-1 / 2},
$$

where $\tau_{H}=12 L_{H}^{4} / D W^{2}$ and $L_{H}=\sqrt{\hbar c / 2 e H}$. It is seen from Eq. (9) that at low fields $\left(L_{N}^{2} \ll D \tau_{H}\right)$ increase in resistance in field $H$ is

$$
\frac{\Delta R_{l o c}(T, H)-\Delta R_{l o c}(T, 0)}{R_{0}} \approx \frac{0.31}{2} \frac{e^{2} R_{\square}^{s t r}}{\hbar W} \frac{L_{N}^{3}}{D \tau_{H}}=\frac{0.31}{2} \frac{e^{2} R_{\square}^{s t r}}{\hbar} \frac{L_{N}^{3} W}{12 L_{H}^{4}} \propto H^{2} / T .
$$

Quadratic dependence of MR on magnetic field for low-field range is clearly seen (Fig. 8). The temperature dependence of MR is difficult to determine exactly enough due to narrow temperature range of the anomaly. We have found, however, that this dependence is strong (inset in Fig. 8) and in the range 1.4-3 K follows (but rather approximately) the 1/T law, as can be expected. It should also be mentioned that the amplitude of MR attributed to 1D WL found in this study $\left(\Delta R(H) / R\right.$ of the order of $\left.10^{-4}\right)$ agrees well with those found for other 1D metal films, including Au films [16, 21 23].

Quantitative comparison with Eq. (10) and obtaining values of $L_{N}$ from it appears to be quite difficult (or even, at first glance, practically impossible) since we actually do not know exact values of two parameters: sheet resistance, $R_{\square}^{\text {str }}$, of $1 \mathrm{D}$ strip and its width W. Macroscopic film resistance $R_{\square}^{\text {exp }} \approx 5.2 \mathrm{k} \Omega$ can be substituted for $R_{\square}^{\text {str }}$, but this can cause some error in calculated values of $L_{N}$ since it is expected that $R_{\square}^{s t r} \ll R_{\square}^{e x p}$. A rough estimate of $W$ can be done taking into account that in 1D films the transition from the 1D to 2D WL behavior of MR takes place when $L_{H}$ with increasing field becomes smaller than width $W$ [16]. It follows from Fig. 7 that in the film studied the transition field, $H_{t r}$, at which the MR anomaly saturates, depends on temperature. The magnitude of $H_{t r}$ grows from $\approx 0.013 \mathrm{~T}$ to $\approx 0.03 \mathrm{~T}$ with decrease in temperature from $T=3 \mathrm{~K}$ to $T=0.38 \mathrm{~K}$, which implies decreasing in $W$ from $\approx 160 \mathrm{~nm}$ to $\approx 100 \mathrm{~nm}$ for this temperature variation (if to take magnetic lengths $L_{H}$ for these values of $H_{t r}$ as values of $W$ ). Decreasing in $W$ with decreasing temperature is not surprising and, furthermore, quite expected in the light of the above-discussed reasons and conditions of forming of 1D percolation structure in quench-condensed ultra-thin films. 
Taking nominally the obtained in the above-mentioned way values of $W$ and $R_{\square}^{\exp } \approx$ $5.2 \mathrm{k} \Omega$, we have calculated $L_{N}$ values at different temperatures with help of Eq. (10) for low-field MR. The result is shown in Fig. 9. It can be seen that in the range 1.5-3 K the calculated values of of $L_{N}$ are proportional to $T^{-0.386}$ which is close enough to theoretical prediction $L_{N}=\left(D \tau_{N}\right)^{1 / 2} \propto T^{-1 / 3}$. Derived values of $L_{N}$ appear to be rather larger than those of length $L_{\varphi}=\left(D \tau_{\varphi}\right)^{1 / 2}$, determined from 2D MR curves in the same range 1.4-3 K (compare Figs. 5 and 9). It is important to mention as well that obtained values of $L_{N}$ exceed the characteristic inhomogeneity scale of the granular film studied, the grain size, which, according to Ref. [9], is between 10 and $20 \mathrm{~nm}$ in quench-condensed ultrathin gold films. This is the necessary condition for observation of WL effects in granular or island films. Unfortunately, it is not possible to find the magnitudes of corresponding relaxation times, $\tau_{N}$ and $\tau_{\varphi}$ since we cannot determine true values of respective electron diffusion coefficients $D$ for the 1D and 2D cases in the inhomogeneous percolating film studied.

It should be noted that calculated values of $L_{N}$ are quite smaller than estimated width $\mathrm{W}$ of inferred 1D strip(s) in the film studied. As it is indicate above, this can be connected with uncertainty in value of $R_{\square}^{s t r}$ in processing data with Eq. (10): $R_{\square}^{\text {str }}$ can be even ten times smaller than the value which is used $(5.2 \mathrm{k} \Omega)$. The uncertainty in $R_{\square}^{\text {str }}$ should not, however, affect appreciably the temperature dependence $L_{N}(T)$, which we have obtained (Fig. 9).

For both, 1D and 2D WL interference effects in the film studied, the phase relaxation lengths obtained seem to saturate going to low temperature (Figs. 5 and 9). The saturation is more pronounced for higher $U_{\text {appl }}$ (Fig. 5) that can be determined by overheating effect. But the saturation persists even at lower $U_{a p p l}$, and this can be determined by 2D inhomogeneous granular structure as suggested in Ref. [24].

Now consider more closely dependence $L_{\varphi}(T)$ obtained from analysis of $\mathrm{MR}$ in $2 \mathrm{D}$ case using Eqs. (44) and (5). In the range 3-15 K, dependence $L_{\varphi} \propto T^{-0.35}$ has been found (Fig. 5). At first glance this seems unexpected since at low temperatures in 2D systems the phase relaxation due to EEI should dominates with rate, $\tau_{e e}^{-1} \propto T$, given by Eq. (3), and $L_{\varphi} \propto T^{-1 / 2}$ as it is usually observed for homogeneous enough gold films (see Ref. [11, 25] and references therein). As was indicated above, the diffusion length of phase relaxation has general temperature dependence $L_{\varphi} \propto T^{-p / 2}$ where index $p$ depends on mechanism of phase relaxation $\left(\tau_{\varphi} \propto T^{-p}\right)$. It is known that in percolating gold films experimental values of 
the index $p$ are often found to be smaller than in homogeneous films. For example, in Refs. [12, 25] dependences $L_{\varphi} \propto T^{-1 / 3}$ and $L_{\varphi} \propto T^{-0.35}$ were found, respectively, with fitting to Eq. (44) for $2 \mathrm{D} \mathrm{WL}$; whereas, $L_{\varphi} \propto T^{-1 / 2}$ is expected for $p=1$. In the first case [12], it was attributed to influence of $1 \mathrm{D}$ regions in the percolating gold film; whereas, in the second case [25], conception of anomalous electron diffusion for inhomogeneous films was involved at the explanation. In any case it is clear that the same phenomenon in the film studied is determined by inhomogeneity of the film.

The data obtained testify that with increasing temperature the film becomes more homogeneous in respect to quantum interference effects: 1D effects in MR disappear above $3 \mathrm{~K}$ (Figs. 7 and 8); $R(T)$ becomes purely logarithmic above $12 \mathrm{~K}$ as expected for $2 \mathrm{D}$ systems (Fig. 3). The dependence $L_{\varphi}(T)$ behaves in the line with this tendency, changing above $15 \mathrm{~K}$ to $L_{\varphi}(T) \propto T^{-1.05}$ (Fig. 5), that corresponds to $p=2.1$. This agrees with results for disordered but homogeneous enough gold films [11], where this has been convincingly attributed to influence of electron-phonon phase relaxation.

In conclusion, we have found that the transport properties of the inhomogeneous ultrathin quench-condensed gold film at low temperature reveal simultaneously indications of the quantum interference 1D and 2D effects of weak localization and electron-electron interaction. With increasing temperature the film behaves as more homogeneous and only 2D effects have been seen. The observed behavior can be explained by inhomogeneous electron transport at the threshold of thickness-controlled metal-insulator transition.

Work was supported in part by program "Structure and Properties of Nanosystems" (Subsection 1.3.) of National Academy of Sciences of Ukraine. 
* belevtsev@ilt.kharkov.ua

[1] E. Abrahams, P. W. Anderson, D. C. Licciardello, and T. V. Ramakrishnan, Phys. Rev. Lett. 42, $673(1979)$.

[2] D. M. Basko, I. L. Aleiner, and B. L. Altshuler, Ann. Phys. 321, 1126 (2006).

[3] V. F. Gantmakher and and V. I. Dolgopolov, Usp. Fiz. Nauk 178, 3 (2008); V. F. Gantmakher and V. I. Dolgopolov, ibid 180, 3 (2010).

[4] B. L. Altshuler and A. G. Aronov, in Electron-Electron Interactions in Disordered Systems, edited by A. L. Efros and M. Pollak, (North-Holland, Amsterdam, 1985), p.1.

[5] B. I. Belevtsev, Usp. Fiz. Nauk 160, 65 (1990) [Sov. Phys. Usp. 33, 36 (1990)].

[6] I. S. Beloborodov, A. V. Lopatin, V. M. Vinokur, and K. B. Efetov, Rev. Mod. Phys 79, 469 (2007).

[7] H. M. Jaeger, D. B. Haviland, and A. M. Goldman, Phys. Rev. B 34, 4920 (1986); N. Marković, C. Christiansen, and A. M. Goldman, Phys. Rev. Lett. 81, 5217 (1998).

[8] B. I. Belevtsev, E. Yu. Belyaev, Yu. F. Komnik, and E. Yu. Kopeichenko, Low Temp. Phys. 23, 724 (1997) [Fiz. Nizk. Temp. 23, 965 (1997)].

[9] K. L. Ekinci and J. M. Valles Jr., Acta Mater. 46, 4549 (1998); Phys. Rev. B 58, 4347 (1998).

[10] A. Palevskii and G. Deutscher, Phys. Rev. B 34, 431 (1986).

[11] B. I. Belevtsev, Yu. F. Komnik, and E. Yu. Beliayev, Phys. Rev. B 58, 8079 (1998).

[12] M. E. Gershenson, P. M. Echternach, H. M. Bozler, A. L. Bogdanov, and B. Nilsson, Phys. Rev. Lett. 74, 446 (1995).

[13] A. V. Butenko, E. I. Bukhshtab, and V. V. Pilipenko, Fiz. Nizk. Temp. 10, 773 (1984) [Sov. J. Low Temp. Phys. 10, 407 (1984)].

[14] S. Wind, M.J. Rooks, V. Chandrasekhar, and D. E. Prober, Phys. Rev. Lett. 57, 633 (1986).

[15] W. D. Williams and N. Giordano, Phys. Rev. B 33, 8146 (1986).

[16] P. M. Echternach, M. E. Gershenson, H. M. Bozler, A. L. Bogdanov, and B. Nilsson, Phys. Rev. B 48, 11516 (1993); ibidem, 50, 5748 (1994).

[17] B. L. Altshuler and A. G. Aronov, Pis'ma Zh. Eksp. Teor. Fiz. 33, 515 (1981) [JETP Lett. 33, 499 (1981)].

[18] B. L. Altshuler, A. G. Aronov, and D. E. Khmelnitskii, J. Phys. C 15, 7367 (1982). 
[19] P. Sheng, Philos. Mag. B 65, 357 (1992).

[20] P. Markoš, Physica B 405, 3029 (2010).

[21] J. J. Lin and N. Giordano, Phys. Rev. B 33, 1519 (1986).

[22] A. P. Heraud, S. P. Beaumont, C. D. W. Wilkinson, P. C. Main, J. R. Owers-Bradley, and L. Eaves, J. Phys. C 20, L249 (1987).

[23] F. Pierre, A. P. Gougam, A. Anthore, H. Pothier, D. Esteve, and Norman O. Birge, Phys. Rev. B 68, 085413 (2003).

[24] A. V. Germanenko, G. M. Minkov, and O. E. Rut, Phys. Rev. B 64, 165404 (2001).

[25] G. Dumpich and A. Carl, Phys. Rev. B 43, 12074 (1991); A. Carl, G. Dumpich, Ch. Buchal, and B. Stritzker, Z. Phys. B 90, 261 (1993). 


\section{Figure captions}

Fig. 1. Temperature behavior of sheet resistance of the gold film studied measured at different magnitudes of the de voltage $\left(U_{\text {appl }}\right)$. The $R_{\square}(T)$ curves are shown for zero magnetic field (filled symbols) and the field $H=4.2 \mathrm{~T}$ (empty symbols).

Fig. 2. (Color online) Dependences of resistance on applied voltage, $U_{\text {appl }}$, in low temperature range. The inset shows $R_{\square} v s U_{\text {appl }}$ at $T=1,36 \mathrm{~K}$ in the region of low voltages.

Fig. 3. Temperature dependence of sheet resistance of the gold film measured at $U_{\text {appl }}=$ $0.2 \mathrm{~V}$ in zero magnetic field and field $H=4.2 \mathrm{~T}$. The $R(T)$ curves follows the logarithmic law above $T \approx 8 \mathrm{~K}$. Below this temperature, deviations, $\Delta R$ from logarithmic growth of resistance with decreasing temperature are developed. Temperature behavior of these lowtemperature deviations is presented in the inset as $\Delta R$ vs $T^{-1 / 2}$.

Fig. 4. Magnetoresistance curves of the film studied measured at applied voltage $U_{\text {appl }}=$ $0.2 \mathrm{~V}$ for magnetic field normal to the film.

Fig. 5. (Color online) Calculated temperature dependence of the phase relaxation length $L_{\varphi}(T)$ in the film obtained using Eq. 4 for different magnitudes of $U_{\text {appl }}$.

Fig. 6. Magnetoresistance curve at $T=1.38 \mathrm{~K}$ and $U_{\text {appl }}=0.2 \mathrm{~V}$. The inset shows enlarged view of low-field part of the curve.

Fig. 7. Temperature evolution of the low-field anomaly of MR attributed to 1D effects in transport properties.

Fig. 8. MR vs. square of magnetic field (at $T=1.38 \mathrm{~K}, U_{\text {appl }}=0.2 \mathrm{~V}$ ). Inset shows temperature dependence of saturation MR in the low-field MR anomaly.

Fig. 9. Temperature dependence of Nyquist length $L_{N}$ calculated using Eq. (10) at $U_{\text {appl }}=3 \mathrm{~V}$. 


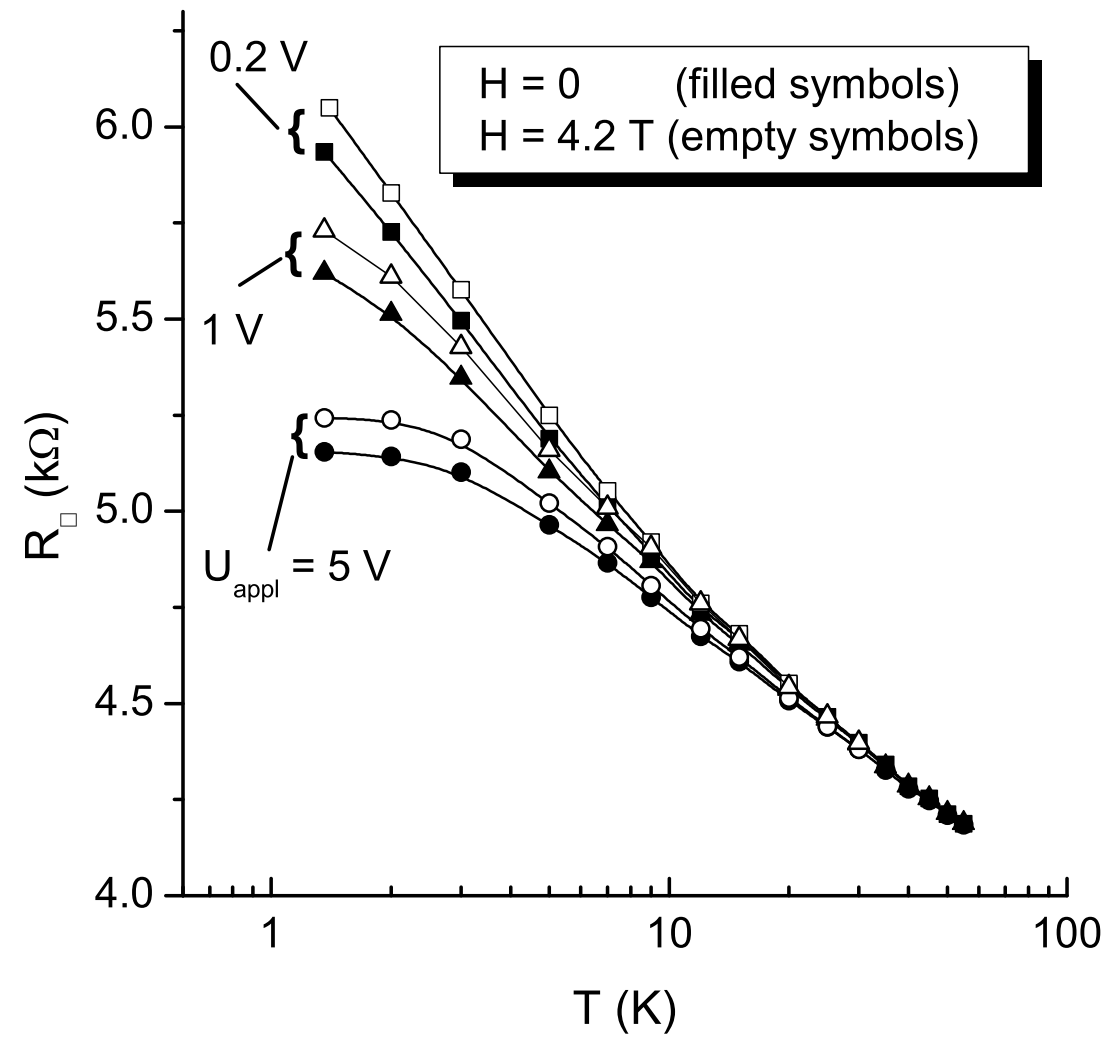

Fig. 1 to paper Beliayev et al. 


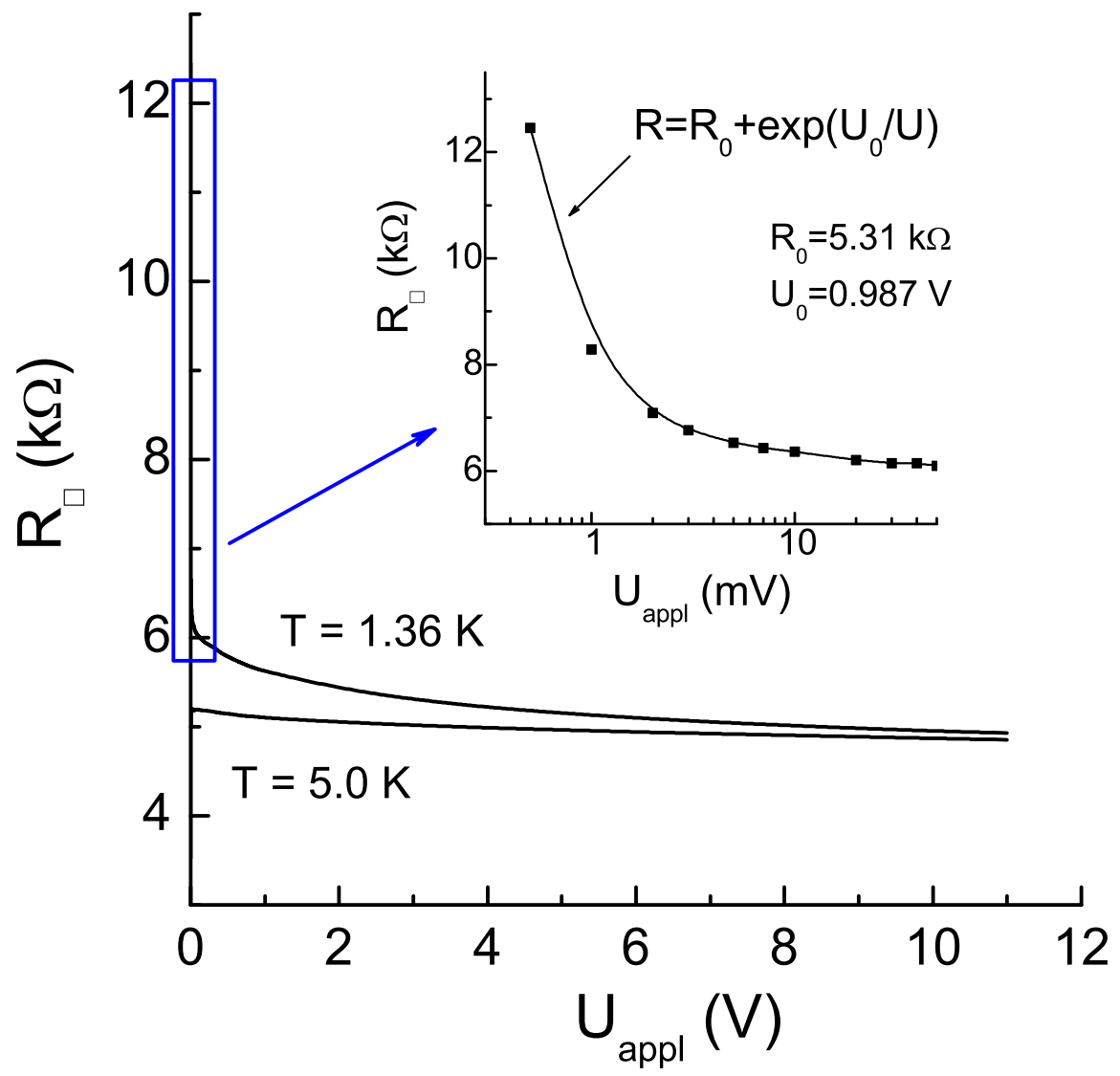

Fig. 2 to paper Beliayev et al. 


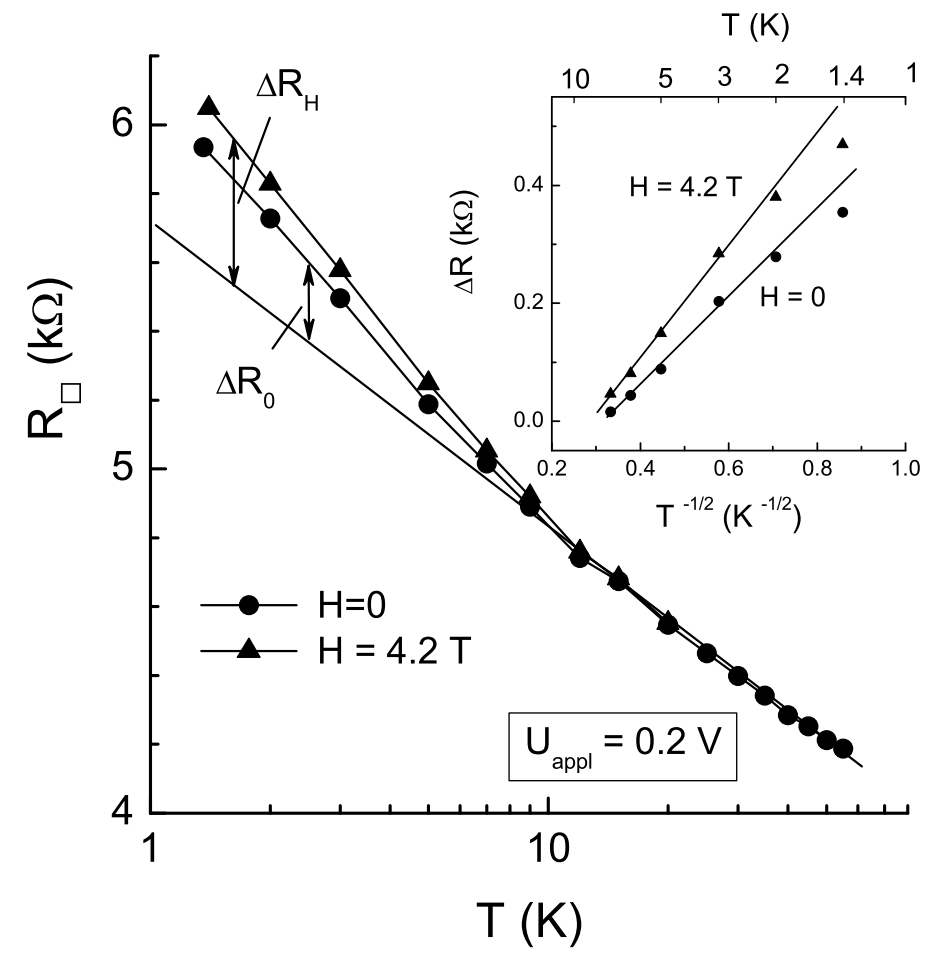

Fig. 3 to paper Beliayev et al. 


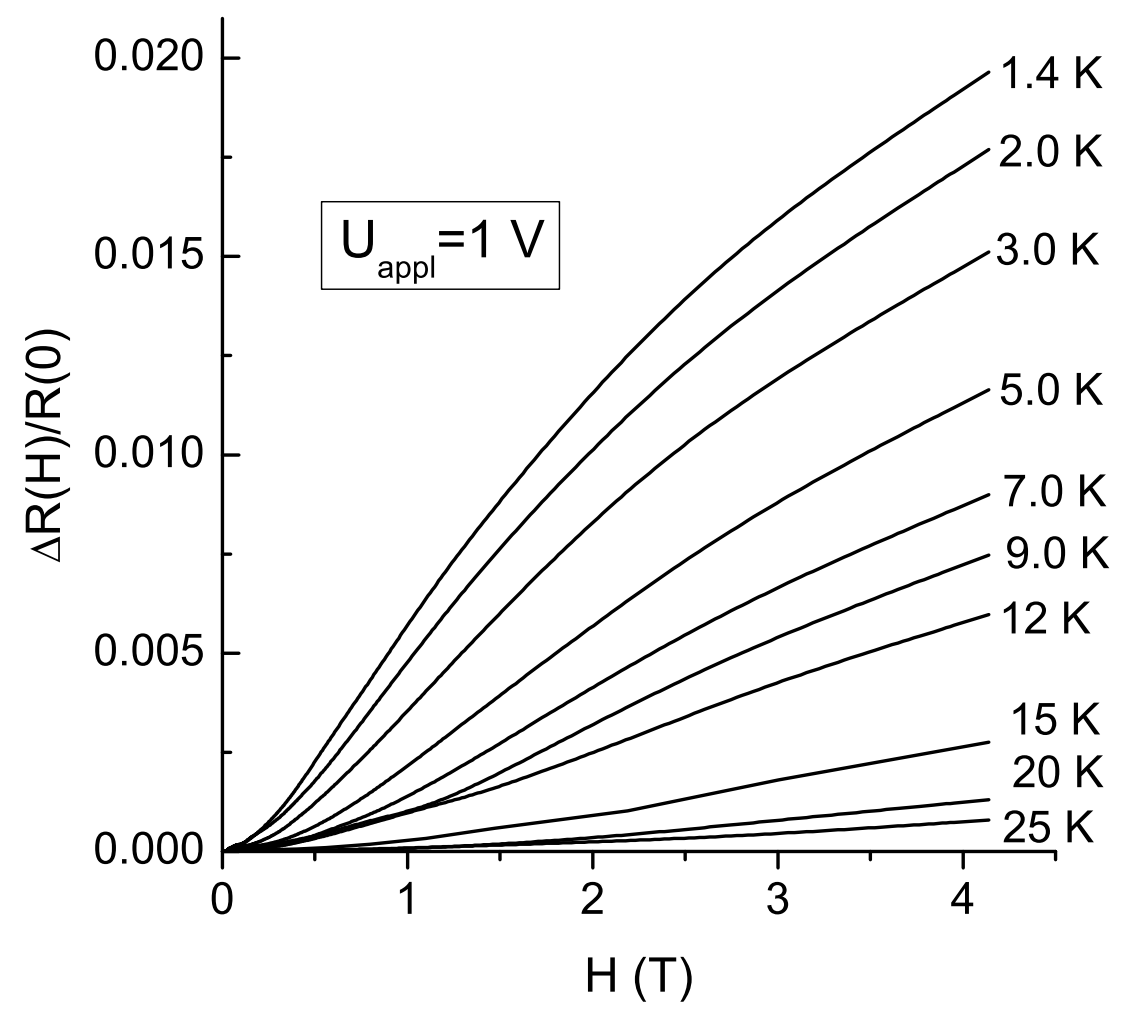

Fig. 4 to paper Beliayev et al. 


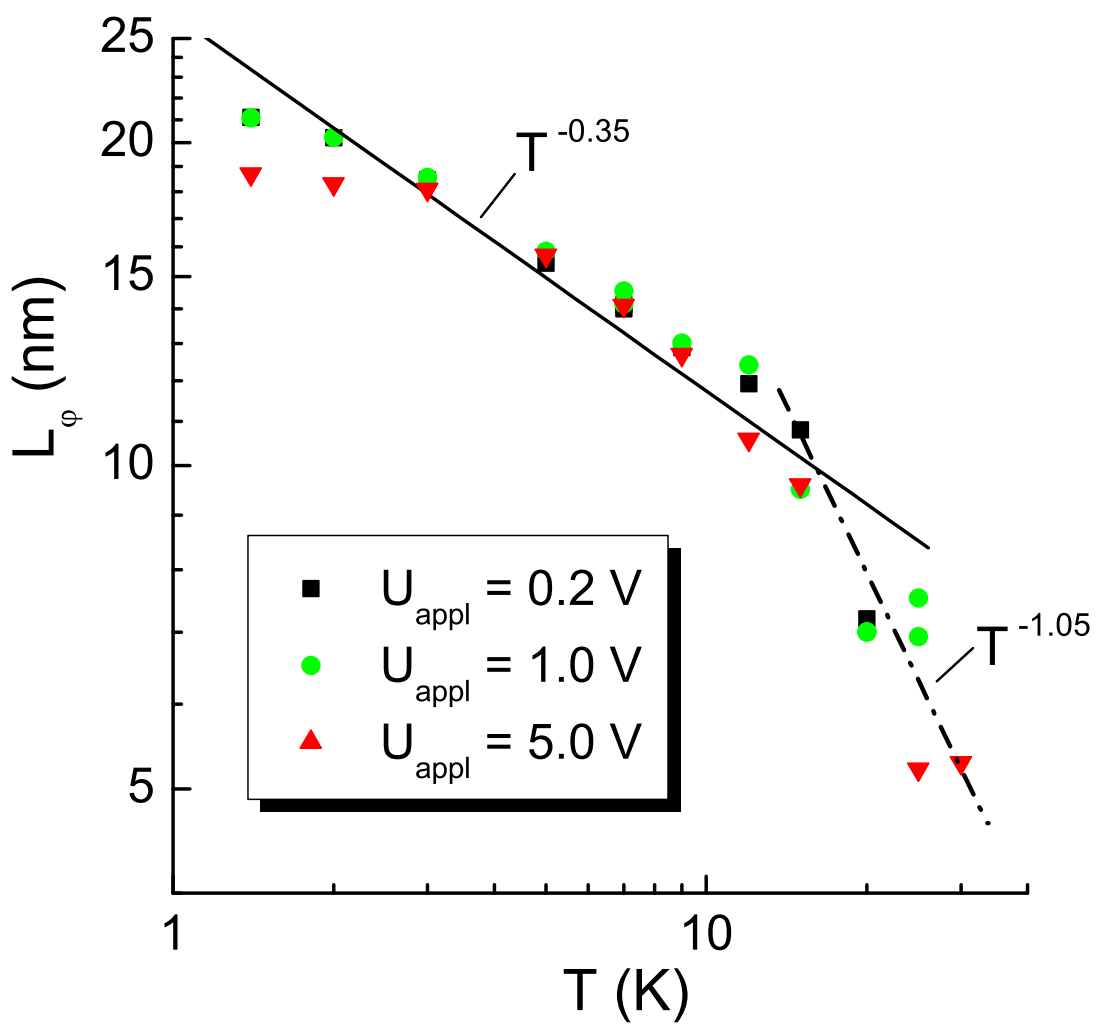

Fig. 5 to paper Beliayev et al. 


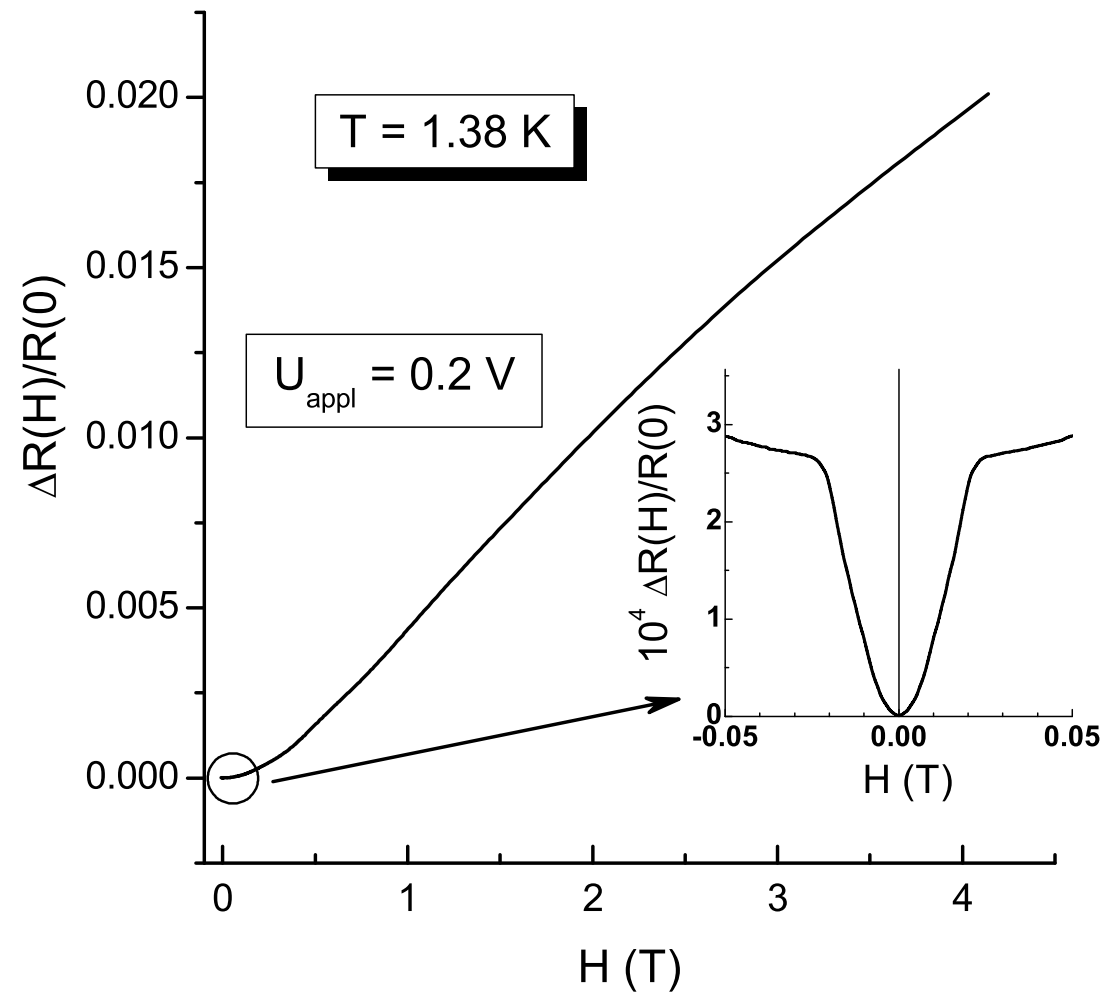

Fig. 6 to paper Beliayev et al. 


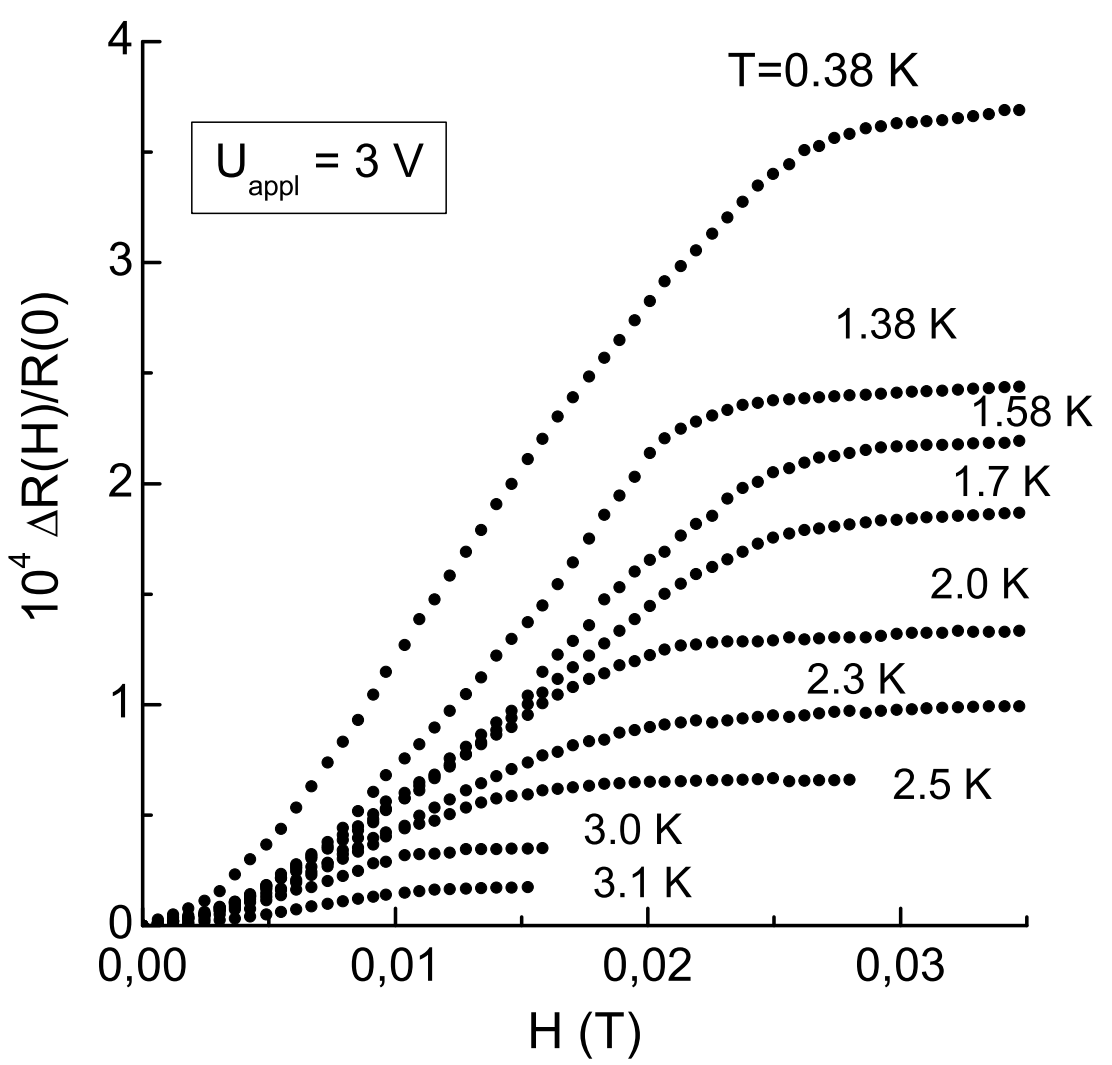

Fig. 7 to paper Beliayev et al. 


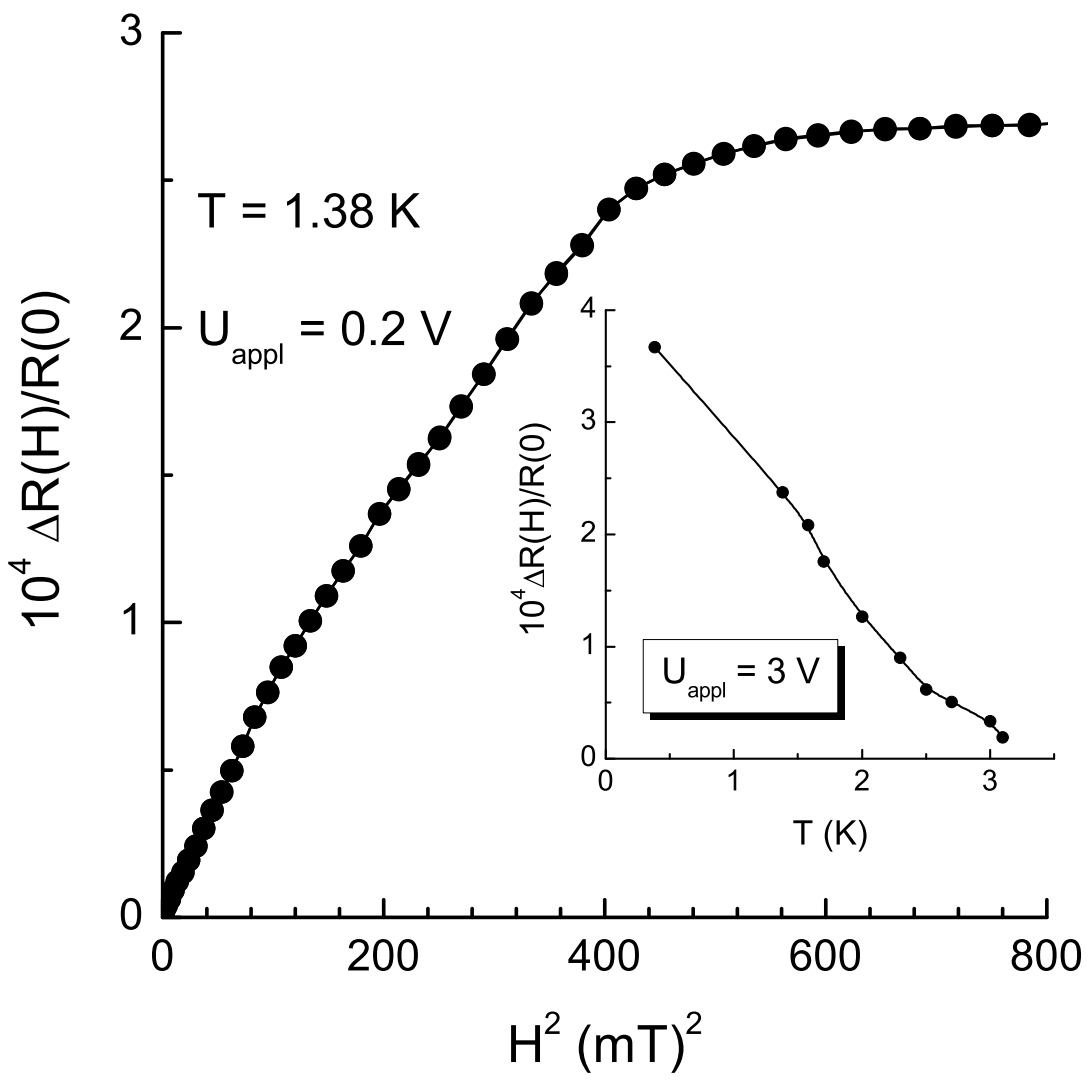

Fig. 8 to paper Beliayev et al. 


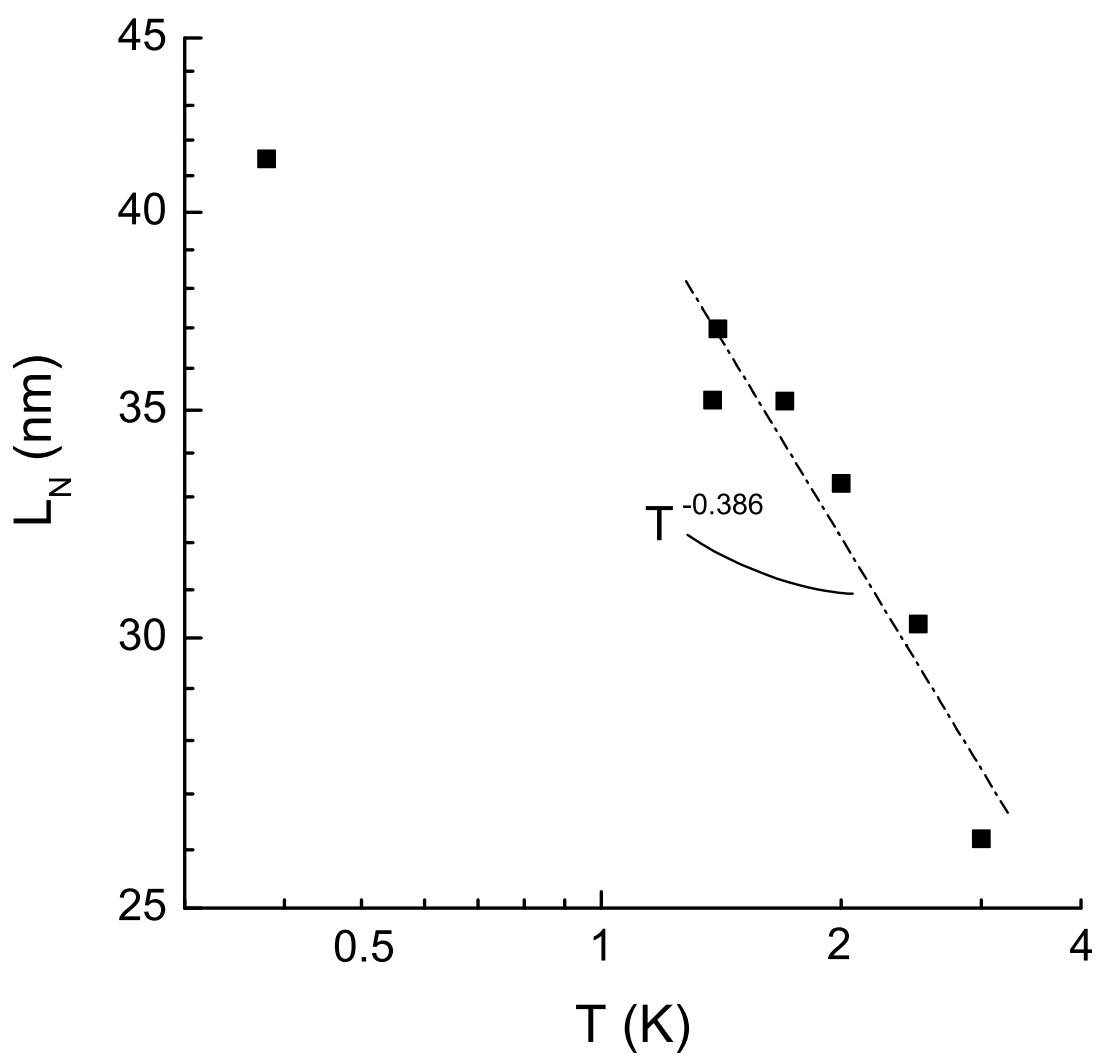

Fig. 9 to paper Beliayev et al. 